\title{
Research and Implementation of mobile context-aware music playlist recommendation system
}

\author{
Qi Shen ${ }^{1, a}$, Ran Wang ${ }^{2, b, ~ * ~}$ \\ ${ }^{1}$ Beijing University of Technology School of Software Engineering, Beijing, 100124, China \\ ${ }^{2}$ Beijing University of Technology School of Software Engineering, Beijing, 100124, China \\ A shenq@bjut.edu.cn, ${ }^{\text {b}} 547066756 @ q q . c o m,{ }^{*}$ corresponding author
}

Keywords: context-aware recommendation; music playlist recommendation; recommendation system

\begin{abstract}
Music recommendation system helps users to find the music they like quickly. This paper proposed a new way to recommend music playlist combined with the user's context and their similar user's music favor. Then research the technology of the mobile context-aware playlist recommendation system in detail and proposed a implement architecture of it.
\end{abstract}

\section{Introduction}

In recent years, with the rapid development and popularization of multimedia technology, Digital music has entered many aspects of people's lives. And in essence, it influences and changes people's multimedia consumption habits. With the continuous expansion of the scale of music database, How to effectively help users find the right music quickly and build a playlist is the main task of the music recommendation system.

The traditional music recommendation system is basically divided into two categories:recommendation system based on collaborative filtering and recommendation system based on content ${ }^{[1]}$. They simply analyze the interests of users or the melody to make a recommendation. And do not analyze the actual environment of the user. In the real world, where we are and what we do now may has a huge influence about our music flavor. Now smart phone is equipped with a variety of sensors. These sensors can be used to predict the user context. And Combined with context information can be recommended more in line with the user's current situation of the music.

\section{Mobile context-aware music playlist recommendation}

Context refers to the additional information that is represented in the user model, Involve "physical context (for example, location, time), context (weather, light and sound intensity) and contextual information (stock quotes, sports scores), personal context (health, mood, planning activities, the social context (team activities, social activities, and who in a room), application context (email, visit the site) and system context (network connectivity status, status of the printer)" and so on information. A music recommendation algorithm based on context awareness in mobile environment is proposed in this paper, is that through the intelligent terminal sensor prediction of user's context, and recommends a series of songs with the context information.

\section{Analyzing playlist title}

There are thousands of users to create playlists on the Internet. The playlist may is a collection about a star or may be users like collection of songs about specfic scenarios. The playlist title of semantic analysis to different playlist classification in accordance with the appropriate context. This paper uses Spotify API to obtain 1000 different users set records for the experimental data, some data format is as follows: 
We assume that a user context space $X=\left\{x_{1}, x_{2}, \ldots, x_{n}\right\}$. Our target is calculated semantic similarity $\mathrm{S}, S \in(0,1)$ between the description item in the Spotify data and ${ }^{X_{i}}$. If $\mathrm{S}$ is close to 1 , on behalf of the playlist more suited to recommend to users in the context. The calculation method is as follows:

1. Split the description item according space. Assuming there are L items after the word segmentation

2. Excluding semantically unrelated words, such as is, the. Get $L^{\prime}$ items

3. For each $d_{l}$ after the word segmentation, get semantic similarity $s_{l}, s_{l} \in(0,1)$ between $d_{l}$ and $x_{i}$ using wordNet query.

4. $S_{i}=\max \left(s_{l}\right)$

\section{Forecasting user context}

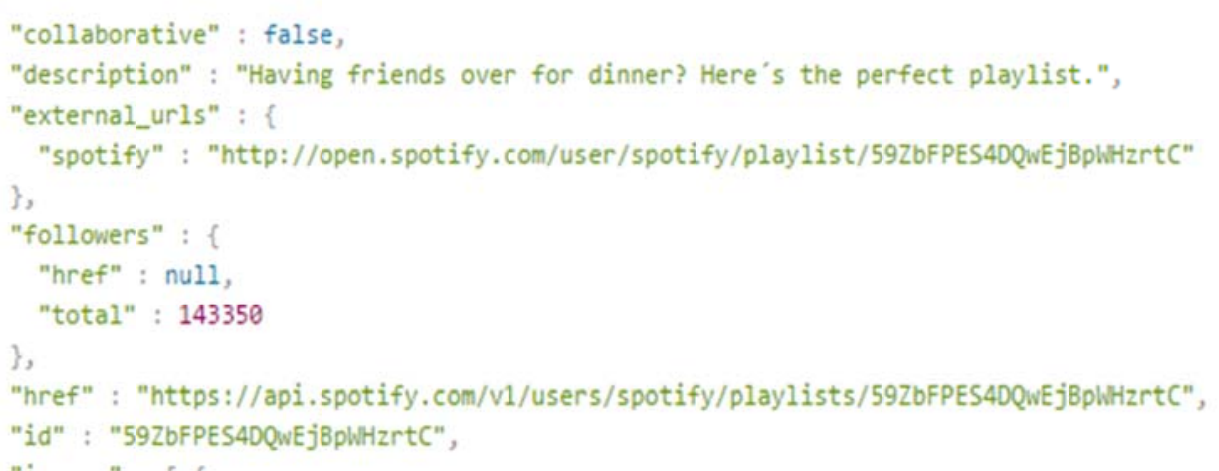

Assume user context $X=\left\{x_{1}, x_{2}, \ldots, x_{m}\right\}, \mathrm{N}$ dimensional data can be obtained from the mobile terminal $D=\left\{d_{1}, d_{2} \mathrm{~K}, d_{n}\right\}$. The formal definition of the forecasting user context problem is, find let $P\left(X_{i} \mid D\right)>P\left(X_{j} \mid D\right) 1 \leq j \leq m, j \neq i$ 。

We use the Android smartphone platform, sensor data obtained through the Android SDK, including accelerometer, gyroscope sensor combined with the user the time, through the analysis of these data, to forecast the user context.

In this paper, the user context is predicted by Naive Bayesian classifier. Assume $X=\{$ walking, running, rest, working $\}$, The feature vector obtained from the Android platform is $D=\left\{d_{a}, d_{g}, d_{t}\right\}, d_{a}, d_{g}, d_{t}$ are Acceleration sensor data, gyro sensor data and time data, According to Bias theorem $P(A \mid B)=P(B \mid A) P(A) / P(B)$ we can get

$P\left(\right.$ walking $\left.\mid d_{a} \times d_{g} \times d_{t}\right)$

$=P\left(d_{a} \mid\right.$ walking $) \times P\left(d_{g} \mid\right.$ walking $) \times P\left(d_{t} \mid\right.$ walking $) \times P($ walking $) / P\left(d_{a}\right) \times P\left(d_{g}\right) \times P\left(d_{t}\right)$

The above probability can be obtained through the sensor data obtained before we get, so we can calculate the maximum value of $P\left(X \mid d_{a} \times d_{g} \times d_{t}\right), X$ is the result of our prediction of user behavior.

\section{Finding similar users}

When the user is in the same context, different users may have different music preferences. If two users in similar semantics playlist used the same song, two users in the same context have similar preferences and define their similar users. In the same semantic collection of songs with the same number of songs, the greater the degree of similarity between users. In the recommendation, will provide users preferred user created a high similarity.

User similarity calculation can be used to calculate the angle of vector cosine. Cosine similarity is used as the cosine of the angle between two vectors in the vector space, which is used to measure 
the difference between the two individuals. The more close to the cosine value of 1 , it shows that the closer the angle of 0 degrees, that is, the more similar the two vectors.

The similarity between the user $\mathrm{A}$ and $\mathrm{B}$ is calculated by the following formula:

$$
\cos (\theta)=\frac{\sum_{i=1}^{n}\left(x_{i} \times y_{i}\right)}{\sqrt{\sum_{i=1}^{n}\left(x_{i}\right)^{2}} \times \sqrt{\sum_{i=1}^{n}\left(y_{i}\right)^{2}}}
$$

Assume user A collect $s_{1}, s_{2}, s_{3}$ in context $x_{1}$, user B collect $s_{1}, s_{2}, s_{4}$ in context $x_{1}$. Then get the vector of $\mathrm{A}$ and $\mathrm{B}\{1,1,1,0\}\{1,1,0,1\}$. So the similar between $\mathrm{A}$ and $\mathrm{B}$ is:

$$
\cos (\theta)=\frac{1 \times 1+1 \times 1+1 \times 0+0 \times 1}{\sqrt{1^{2}+1^{2}+1^{2}+0^{2}} \times \sqrt{1^{2}+1^{2}+0^{2}+1^{2}}}=\frac{2}{3}
$$

\section{Architecture of mobile context-aware playlist recommendation}

\section{Physical architecture}

In order to achieve a context recommendation system is put forward in this paper, We need a smart terminal to collect user data to forecast user's context. And There are lots of playlist on the internet, We need a offline ML cluster to claw and analysis the relationship of data. Because some operations are very time consuming, the offline ML cluster is responsible for find user's similar user and analysis playlist titile at regular time. In that period, the user's relationship will not change. The realtime recommendation cluster is responsible for recommending playlists combined with the result of offline ML.the physical structure as shown in Fig.1

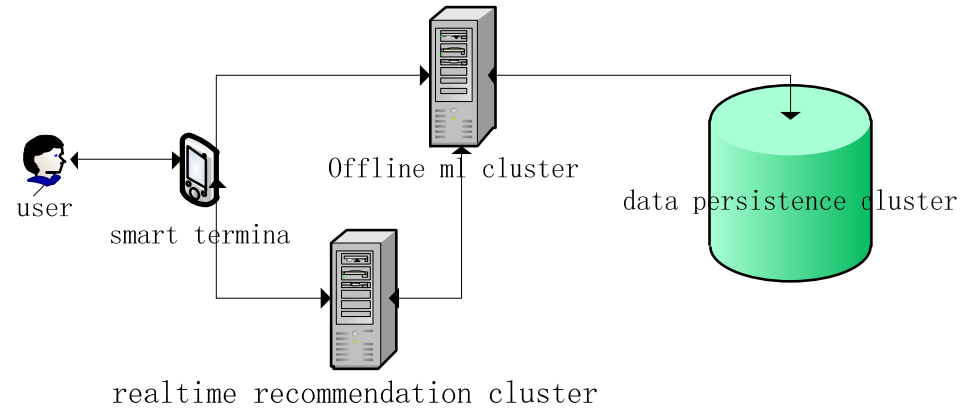

Fig.1 physical architecture

\section{Logical architecture}

In this paper, the realization of context playlist recommendation system mainly includes data collection module, data storage module, playlist Title Semantic analysis module, to find similar users module, real-time recommendation result generation module, audio module and the relationship between these modules as shown in Fig.2: 


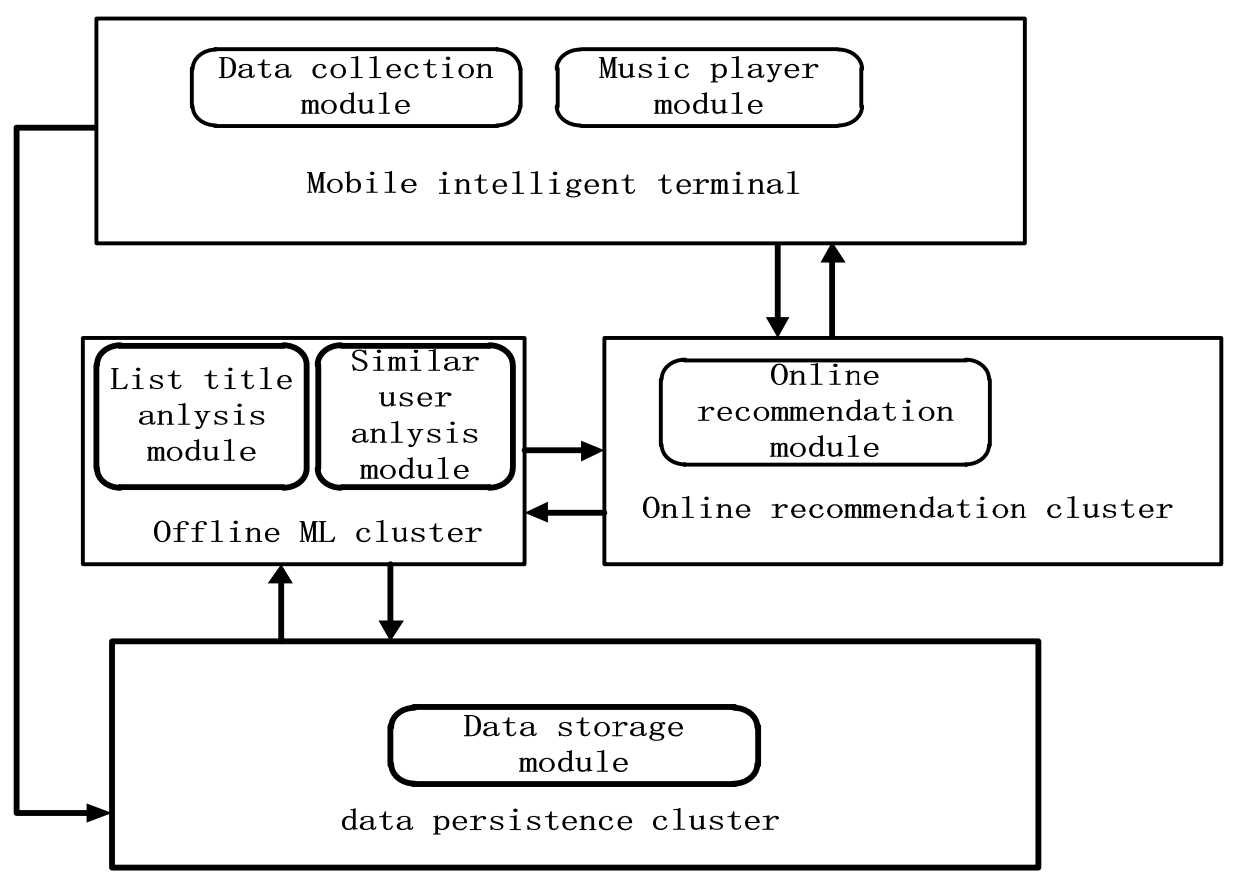

Fig.2 Logical architecture

Data collection module and Music player module are settled in mobile terminal. Data collection module is responsible for collecting sensor data and save the data in data storage module. Then transfer sensor data to online recommendation module to forecast user context. Music player module provides playing the recommend result, skipping and other functions.

Playlist title analysis module and similar user analysis module are settled in offline machine learning cluster. Playlist title analysis module is responsible for clawing playlist data from Spotify, and analyzing playlist title to classify them(as shown in 3.1). Similar user analysis module is responsible for analyzing user data and get similar user of users (as shown in 3.3)。

Online recommendation module is settled in online recommendation cluster. It is responsible for receiving sensor data from data collection module to forecast user context (as shown in 3.2) and combine the result of offline machine learning to generate the recommendation playlist.

Data storage module is settled in data persistence cluster. It is responsible for saving and loading data of the recommendation system.

\section{The process of recommendation}

The process of mobile context-aware music list recommendation is shown in Fig.3: 


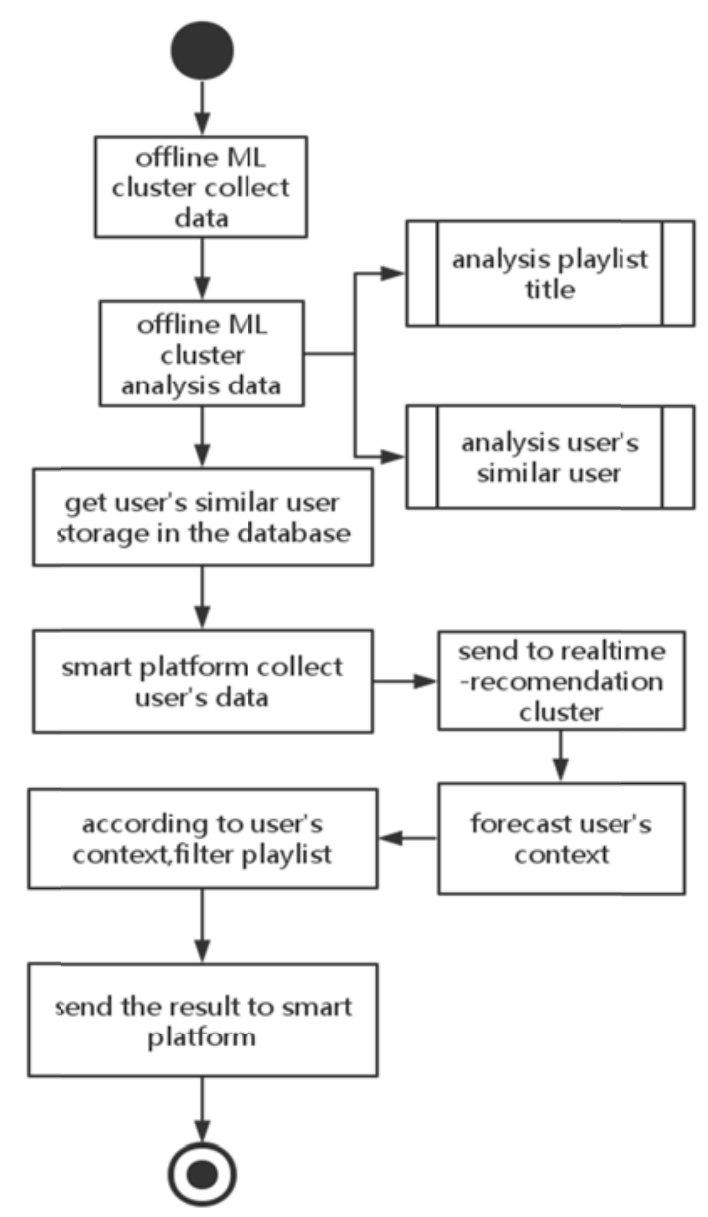

Fig..3 The process of recommendation

\section{System implementation and conclusion}

We have make a prototype as shown in Fig.4. When user entry the app, it will collect sensor data automatic to forecast user context. Then recommend playlists combined with context.

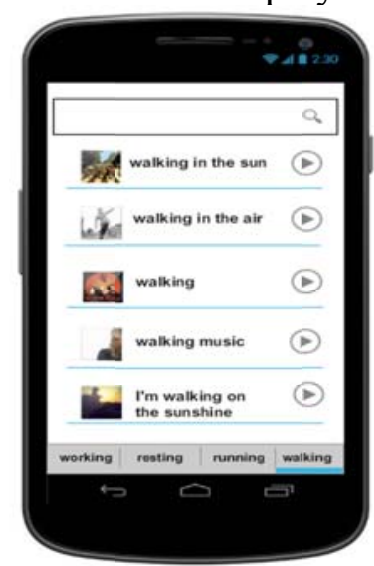

Fig. 4 system prototype

For comparison the effect of recommendation. We set 3 modes in the prototype. Recommended Spotify hot list mode $(\mathrm{m} 1)$, Only recommend similar users favorite mode $(\mathrm{m} 2)$ and mobile context-aware playlist recommendation (m3). We collect the satifisfied of three different mode. The result is shown in Fig.5. 


\section{satisfied}

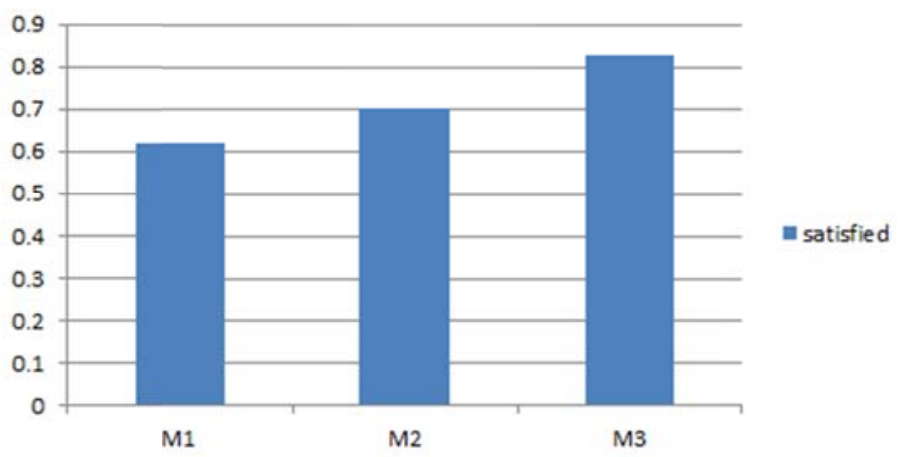

Fig.5 Comparison results

We can see that $\mathrm{m} 3$ mode improve the satisfied of the recommendation.

\section{Summary}

First, this paper proposed a new way to recommend playlist and researched the related technology of mobile context-aware playlist recommendation. Then describe architecture of mobile context-aware playlist recommendation and explain every module in the system. At the end, this paper gives a system prototype and a conclusion based on the prototype. We can see the prototype is going well and improve the effect of recommendation.

\section{Reference}

[1] Wang Licai, Meng Xiangwu, Zhang Yujie. context aware recommendation system [J].Chinese Journal of software, 2012,01:1-20.

[2] Chen Yaqian. Music recommendation system and related technology research [J]. computer engineering and application, 2012,18:9-16+47.

[3] Lin Hongfei, Li Ruimin, Yan Jun. Based on the user - label - Project semantic mining personalized music recommendation [J]. computer research and development, 2014,10:2270-2276.

[4] Tan Xueqing, He Shan. A review of the research on music personalized recommendation system [J]. modern library and information technology, 2014,09:22-32.

[5] Goldberg K, Roeder T, Gupta D, Perkins C. Eigentaste: A constant time collaborative filtering algorithm[J]. Information Retrieval. 2001,4(2):133-51. 